Medici, Ch. Estrategias para el aborto legal: la incidencia internacional. Argentina (2000-2017). Derecho y Ciencias Sociales. Mayo - Octubre 2020. No 23. Pgs 70-92 ISNN 1852-2971 Instituto de Cultura Jurídica y Maestría en Sociología Jurídica. FCJ y S. UNLP

\title{
Estrategias para el aborto legal: la incidencia internacional. Argentina (2000-2017)
}

Strategies for the implementation of legal abortion in Argentina: international advocacy (20002017)

\section{Chantal Medici}

\section{Resumen:}

A partir del nuevo milenio la organización en torno a la demanda de la legalización del aborto en Argentina cobró una importancia sin precedentes. Los feminismos y las organizaciones de Derechos Humanos que se constituyeron como sus aliadas desplegaron variadas estrategias. Entre ellas se destacó la incidencia en organismos supranacionales, como los Comités de las Naciones Unidas y las Conferencias Regionales de la Mujer de la CEPAL, que en respuesta emitieron importantes resoluciones y documentos relativos a las políticas públicas de nuestro país en la materia. Este documento resume el trabajo político de las organizaciones profesionalizadas y las respuestas de los organismos que resultaron en la construcción de un marco más favorable para políticas públicas que garanticen el acceso al aborto legal.

Palaras clave: Argentina - aborto - incidencia - internacional - organismos - internacionales

\begin{abstract}
:
Demand for legalization of abortion has gained unprecedented importance in Argentina since the beginning of the XXI century, as feminism and human rights organizations performed various strategies. Among them, impact on supranational organizations, such as United Nations Committees and the Regional Women's Conferences of ECLAC was outstanding. This document summarizes the actions performed by feminist and human rights organizations and the responses of international organizations that created a favorable frame for public policies that guarantee the access to this right.
\end{abstract}

Key words: Argentina - Abortion -international - impact - organizations

\footnotetext{
- Chantal Medici es socióloga (Universidad de Buenos Aires [UBA]) y Magíster en Políticas Públicas y Gerenciamiento del Desarrollo (Universidad Nacional de San Martín [UNSAM]y Universidad de Georgetown [GU]). Es investigadora en el Centro de Estudios sobre Democratización y Derechos Humanos (CEDEHUUNSAM) en el Grupo de Estudios sobre Movilización y Participación (Centro de Investigación para los trabajadores [CITRA]- CONICET). Mail: chantalmedici2@gmail.com
} 
Medici, Ch. Estrategias para el aborto legal: la incidencia internacional. Argentina (2000-2017). Derecho y Ciencias Sociales. Mayo - Octubre 2020. No 23. Pgs 70-92 ISNN 1852-2971 Instituto de Cultura Jurídica y Maestría en

Sociología Jurídica. FCJ y S. UNLP

Estrategias para el aborto legal: la incidencia internacional. Argentina (2000-2017)

Chantal Medici

\section{Introducción}

El reciente debate de la legalización del aborto en el Congreso argentino, en un marco de tres años consecutivos de masivas manifestaciones y paros de mujeres (Iglesias, 4 de junio de 2015; "Miles de personas marcharon", 2017; "Paro Internacional de Mujeres", 2018) puso en esta demanda por primera vez el centro de la agenda. De todas formas, las expresiones más visibles de movilización tienen sustento en una construcción política de larga data.

La labor de los feminismos sobre este tema comenzó en Argentina de los años 80, pero cobró especial impulso a partir del nuevo milenio (Di Marco, 2011: 280-281). Desde entonces se desplegaron diversas estrategias que van desde la creación de redes de organizaciones de base hasta la incidencia en organismos supranacionales y el litigio estratégico.

El presente trabajo, que se ocupa solamente de estas dos últimas formas de acción, es una presentación parcial de una investigación realizada en el marco de una tesis de maestría en la que se indagaron estos variados tipos de activismo puestos en marcha entre los años 2004 y 2017 para el cumplimiento del aborto legal y para la legalización o despenalización de esta práctica.

La metodología combinó la revisión documental en los repositorios del Alto Comisionado para los Derechos Humanos y entrevistas a referentes de las organizaciones cuya acción fue identificada como clave en el proceso. Se incluyen aquellas tomadas a Marta Alanis, dirigente de Católicas por el Derecho a Decidir (CDD), Felicitas Rossi, ex integrante de la Asociación por los Derechos Civiles (ADC), Edurne Cárdenas, miembro del Centro de Estudios Legales y Sociales (CELS), a una investigadora adjunta del Centro de Estudios de Estado y Sociedad (CEDES) y Cristina Zurutuza del Comité de América Latina y El Caribe para la Defensa de los Derechos de la Mujer (CLADEM).

En el primer apartado se resume la situación legislación relativa a la interrupción del embarazo, las contradicciones del abordaje estatal y sus implicancias sociales. Luego se resume la movilización por la legalización del aborto en las últimas décadas y se presentan los principales actores y organizaciones que participaron en la incidencia internacional. Más adelante se presentan las principales acciones de incidencia internacional estudiadas, que son los litigios internacionales y la participación en las reuniones de los Comités de las Naciones Unidas (ONU). 
Medici, Ch. Estrategias para el aborto legal: la incidencia internacional. Argentina (2000-2017). Derecho y Ciencias Sociales. Mayo - Octubre 2020. No 23. Pgs 70-92 ISNN 1852-2971 Instituto de Cultura Jurídica y Maestría en Sociología Jurídica. FCJ y S. UNLP

Respecto a esta última se resumen las Recomendaciones y Observaciones Generales de los organismos para el Estado argentino producto de esas reuniones. Finalmente, Se resume el fallo "F.A.L. s/ medida autosatisfactiva", y se ofrece un detalle de las referencias que hace de estos dictámenes.

\section{El aborto en Argentina}

Al igual que en gran parte de los países de América latina, en Argentina el aborto está regulado a partir de un modelo de causales o indicaciones ${ }^{1}$. El Código Penal considera que la práctica no es punible si se realiza "con el fin de evitar un peligro para la vida o la salud de la madre" o si "el embarazo proviene de una violación o de un atentado al pudor cometido sobre una mujer idiota o demente" (Código Penal Argentino, art. 86).

A pesar de las leyes restrictivas, diversos estudios indicaron que la realización clandestina de estas intervenciones es masiva en Argentina. Estimaciones hechas sobre el año 2004 calcularon que en el país se inducen un promedio de entre 486.000 y 552.000 de abortos al año (Mario y Pantelides, 2009: 112).

Sin embargo, históricamente las mujeres y personas con capacidad de gestar no pudieron acceder a los permisos que concede la ley. Hasta hace pocos años atrás, varias investigaciones (Chiarotti, 2006; Motta y Rodríguez, 2000) señalaban que, al solicitarse un aborto legal en un hospital, a menudo el personal médico desconocía estos derechos y podía entonces negarse a realizarlo (Gogna, Romero, Ramos, Petracci y Szulik, 2002). También repetidamente se requería la participación del comité de ética de la institución y/o una autorización judicial que no es indicada por la ley, lo cual dilataba los plazos y, en consecuencia, frecuentemente derivaba en una declinación del pedido ${ }^{2}$.

Cuando se solicitaba el permiso a la magistratura, esta solía indicar que no era necesario, dado que su rol consistía en manifestarse sobre las prácticas ya realizadas. (Motta y Rodríguez, 2000:

\footnotetext{
${ }^{1}$ Paola Bergallo discute con aquellas posiciones que exponen la legislación sobre la práctica como si estuviera agrupada en dos extremos, uno que la prohíbe y otro que la permite totalmente. Señala que los países adoptaron muy diversas regulaciones o bien optaron por incidir en el fenómeno por medio de políticas distintas a la punición. Los estudios identifican dos tipos de legislación liberalizadora del aborto: por indicaciones o causales, o por plazos. En el primer modelo, tal como sucede en muchos países latinoamericanos, se despenaliza la interrupción de embarazos en ciertas circunstancias, por ejemplo, si se trata del producto de un abuso o que implique riesgos a la vida o salud de quien gesta. En el modelo de plazos se despenaliza el procedimiento en un período de la gestación, estipulado por semanas o trimestres (Bergallo, 2011: 5-7).

${ }^{2}$ Algunos estudios cualitativos insinúan que la falta de celeridad y la multitud de trámites administrativos pueden inducir a las mujeres a abandonar el pedido de realización del aborto, en ocasiones debido a que la situación se prolonga más allá del plazo recomendado para realizarlo en condiciones seguras (Irraizábal, 2015).
} 
Medici, Ch. Estrategias para el aborto legal: la incidencia internacional. Argentina (2000-2017). Derecho y Ciencias Sociales. Mayo - Octubre 2020. № 23. Pgs 70-92 ISNN 1852-2971 Instituto de Cultura Jurídica y Maestría en Sociología Jurídica. FCJ y S. UNLP

15). En ocasiones podía asimismo expedirse en contra de la intervención, dado que existieron posturas que interpretaron la normativa de manera restrictiva o plantearon la inconstitucionalidad del artículo 86.

Por un lado, algunos sectores entendían que el Código Penal establecía la legalidad del aborto practicado sólo sobre mujeres discapacitadas. También se debatió si era necesario probar un determinado nivel de riesgo para la vida o salud de la gestante. (Ferrante, 2010: 365).

Otras voces postularon que el artículo era contrario al derecho a la vida consagrado en diversos tratados y convenciones internacionales a las que se dio jerarquía constitucional a partir de la reforma de 1994, principalmente la Convención Americana de los Derechos Humanos (art. 4.1), la Convención de los Derechos del Niño (art. 6) ${ }^{3}$. A menudo se interpretó también el art. 75 inc. 23 de la Constitución, que encomienda al Congreso "dictar un régimen de seguridad social especial e integral en protección del niño en situación de desamparo, desde el embarazo hasta la finalización del período de enseñanza elemental, y de la madre durante el embarazo y el tiempo de lactancia" como un fundamento para la penalización del aborto en todos los casos (Faerman, 2008).

A pesar de la importancia de la cuestión, primó el silencio sobre la inaccesibilidad del aborto legal durante todo el siglo XX. A partir del nuevo milenio se conocieron una serie de casos en que el acceso a este derecho obstaculizado por el personal estatal, lo cual echó luz sobre un problema largamente ignorado. Uno de ellos fue el de "L.M.R." ${ }^{4}$, joven discapacitada que procuró interrumpir su embarazo producto de un abuso sexual, que devino en un litigio ante el Comité de Derechos Humanos de las Naciones Unidas, y resultó en una sanción para el Estado Argentino, como se detalla más abajo. Otro fue el de “A.M.A.”, quien murió de cáncer al serle denegadas tanto la interrupción del embarazo como la quimioterapia, que es incompatible con la gravidez". Fue emblemático asimismo el incidente conocido como "C.P.d.P., A.K. s/ Autorización", en que se autorizó a realizar la práctica, luego de largas audiencias, a una mujer cuya gestación era peligrosa para su vida por padecer una grave afección cardíaca. Se difundieron episodios en que niñas y adolescentes, incluso de 12 o 13 años, enfrentaron una variedad de

\footnotetext{
${ }^{3}$ La Convención Americana sobre Derechos Humanos en su artículo 4.1 establece que "toda persona tiene derecho a que se respete su vida. Este derecho estará protegido por la ley y, en general, a partir del momento de la concepción. Nadie puede ser privado de la vida arbitrariamente". La Convención de los Derechos del Niño en su artículo 6 indica que "Los Estados parte reconocen que todo niño tiene derecho intrínseco a la vida".

${ }^{4}$ Se utilizan siglas en lugar de nombres completos para proteger la identidad de las mujeres.

${ }^{5}$ La situación dio lugar a la acción denominada "Requerimiento de Instrucción Fiscal № 1 referido a la muerte de A.M.A.”.
} 
Medici, Ch. Estrategias para el aborto legal: la incidencia internacional. Argentina (2000-2017). Derecho y Ciencias Sociales. Mayo - Octubre 2020. № 23. Pgs 70-92 ISNN 1852-2971 Instituto de Cultura Jurídica y Maestría en

Sociología Jurídica. FCJ y S. UNLP

obstáculos burocráticos y violencia institucional para acceder al derecho, o no pudieron hacerlo ${ }^{6}$. La difusión de estas situaciones abrió paso al debate sobre el tema, que entró definitivamente a la agenda pública y cobró peso progresivamente.

\section{Las demandas de la legalización e implementación del aborto legal y sus principales actores}

\subsection{La Campaña por el Aborto Legal, Seguro y Gratuito}

Promediando la primera década del siglo XXI el activismo en torno a las demandas relacionadas con la liberalización del aborto, ya sea la despenalización/legalización como la correcta implementación de los derechos adquiridos, avanzó considerablemente.

En el año 2005 se creó la Campaña Nacional por el Derecho al Aborto Legal, Seguro y Gratuito (en adelante, La Campaña). En la actualidad, La Campaña es un colectivo compuesto por 305 grupos, organizaciones y personalidades vinculadas a los Derechos Humanos de todo el país (La Campaña, s/f), que se convirtió en el principal impulsor de la legalización del aborto en Argentina. Esta red fue responsable de la presentación de proyectos de ley de interrupción voluntaria del embarazo en seis ocasiones consecutivas (2007, 2010, 2012, 2014, 2016 y 2018). El apoyo parlamentario a esta iniciativa creció de manera discontinuada a lo largo de una década, como lo demuestra el número de legisladoras y legisladores que respaldaron la propuesta año a año (22 en 2006; 51 en 2010; 60 en 2012 y 67 en 2014; 35 en 2016 y 71 en 2018) (Tabbush et al., 2016; Campaña por el Aborto Legal, Seguro y Gratuito, 2016; Roffo, 6 de marzo de 2018). Finalmente, se logró media sanción del proyecto de ley en el año 2018, pero la iniciativa fue derrotada en la Cámara Alta.

A lo largo de estos años se realizó constantemente lobby parlamentario y las presentaciones fueron acompañadas por una multiplicidad de acciones de incidencia. Poco a poco el aborto se fue haciendo un lugar en la agenda política.

[“(...) se daba la discusión política y se hacía incidencia en partidos políticos y diputados, y se hacía cabildeo con diputados (...) se hacía una conferencia de prensa, con presencia de diputados/as, y eso trae también prensa, se hicieron seminarios en el Congreso (...). Yo creo que una gran catalizadora de todo este tema fue La Campaña, y

\footnotetext{
${ }^{6}$ Algunos de estos casos se encuentran sintetizados en el documento "Foro: las acciones de La Campaña por el Aborto Legal, Seguro y Gratuito ante Aborto no Punible", editado por La Campaña por el Aborto Legal, Seguro y Gratuito (2009).
} 
Medici, Ch. Estrategias para el aborto legal: la incidencia internacional. Argentina (2000-2017). Derecho y Ciencias Sociales. Mayo - Octubre 2020. No 23. Pgs 70-92 ISNN 1852-2971 Instituto de Cultura Jurídica y Maestría en

Sociología Jurídica. FCJ y S. UNLP

lo es, que nace en el 2005 y en el 2007 (...). La otra cosa fuerte, se hacían marchas y acciones callejeras cada vez que se presentaba el proyecto (...)’] (Entrevista a C. Zurutuza - CLADEM).

Desde el año 2006, La Campaña comenzó también a acompañar a quienes veían denegado su acceso a la práctica en los servicios estatales. La extensa red de organizaciones a menudo permitió tomar contacto con las familias y guiarlas para salvar la variedad de obstáculos interpuestos por diferentes agentes públicos. El colectivo desplegó todo tipo de maniobras y articulaciones para hacer efectivo el derecho, desde la asesoría legal de las solicitantes, la realización de movilizaciones y declaraciones, la coordinación con actores públicos, hasta la búsqueda de profesionales que tuvieran voluntad de realizar la intervención ${ }^{7}$ (Campaña por el Derecho al Aborto Legal, Seguro y Gratuito, 2009).

\subsection{Los grupos académicos}

En forma paralela a este trabajo, el grupo CEDES emprendió la labor de generar de evidencia científica y argumentos jurídicos para la ampliación del acceso al derecho. Desde hacía ya algunos años, CEDES producía información epidemiológica, sociológica y médica sobre el tema, cuando en 2007 comenzó un proyecto de visibilización del aborto legal y de creación de fundamentos legales. Para esta iniciativa se convocó a expertas como Paola Bergallo y Agustina Ramón Michel, quienes generaron un diálogo con eminencias académicas del derecho.

A partir de la idea de que en América Latina predominan los modelos de regulación del aborto por causales y no por plazos, se decidió trabajar en el cumplimiento de la ley existente, desde la construcción de una interpretación jurídica que considerara legal la práctica en variadas circunstancias.

[“(...) nos pusimos en la labor de generar material jurídico, no había, no había nada, había una discusión vieja de penalistas si era demente o no y en esos términos (...), ahi empezamos un trabajo de visibilizar el aborto no punible, es decir que en Argentina había causales de despenalización del aborto pero que había ocurrido una penalización del facto durante casi 80 o 90 años (...) Una de las primeras acciones que hicimos fue buscar un entorno jurídico que nos acompañe (...) Toda esa comunidad no tenía una posición (...) Entonces era una vía de entrada a ampliar"] (Entrevista a investigadora adjunta-CEDES).

\footnotetext{
${ }^{7}$ Los casos acompañados se describen en el documento Campaña por el Aborto Legal, Seguro y Gratuito ante Aborto no Punible (2009).
} 
Medici, Ch. Estrategias para el aborto legal: la incidencia internacional. Argentina (2000-2017). Derecho y Ciencias Sociales. Mayo - Octubre 2020. № 23. Pgs 70-92 ISNN 1852-2971 Instituto de Cultura Jurídica y Maestría en

Sociología Jurídica. FCJ y S. UNLP

Este equipo y las personalidades académicas convocadas elaboraron argumentos jurídicos basados en principios clásicos de la teoría del derecho que luego fueron utilizados en litigios y contribuyeron a construir una nueva perspectiva legal sobre el tema.

["La discusión era entonces a través de uso de principios clásicos del derecho constitucional liberal, el principio de legalidad, no podés ampliar la penalización, es un permiso en el Código Penal, lo tenés que interpretar ampliamente, entonces no dice riesgo grave, no es grave, sí eran argumentos que buscaban ser muy mainstream (...)"] (Entrevista a investigadora adjuntaCEDES).

\subsection{Los organismos de derechos humanos}

Luego de los primeros esfuerzos por instalar el asunto en la agenda política se involucraron también organismos de derechos humanos como el CELS, ADC y más adelante Amnistía Internacional Argentina. Estas dos primeras organizaciones se sumaron a la creación de argumentos jurídicos, litigios estratégicos y la producción de informes sombra en alianza con otras organizaciones.

[“(...) nosotros trabajamos en dos sentidos... tres... Una primera línea es la de generar argumentos jurídicos desde el derecho internacional de los Derechos Humanos, para aportar a los debates legislativos y a los debates sobre el tema en general. Una segunda línea es la línea del litigio estratégico, en el que el CELS trabaja con otras organizaciones en alianza (...). Hay una tercera línea (...), que es nuestro trabajo siempre en alianza llevando este trabajo a los órganos de las Naciones Unidas. (...) yo creo que en la última década es increíble la producción nacional de argumentos (...)”] (Entrevista a E. Cárdenas - CELS).

En el año 2012, CELS se unió además a La Campaña y enmarcó su tarea en esta alianza. Al mismo tiempo, continuó actuando por la defensa del derecho contemplado en el art. 86, también en conjunto con las otras organizaciones que incluían este tema en su agenda.

["El CELS se integra a Campaña Nacional por el Derecho al Aborto Legal, Seguro y Gratuito en el año 2012, y entonces, por ejemplo, estas acciones de generar argumentos para la legalización del aborto entran dentro de esa... de esa gran estrategia, pero una segunda estrategia, un segundo objetivo que alimenta el objetivo más general es el de garantizar el derecho a los abortos que son legales"] (Entrevista a E. Cárdenas - CELS).

Prácticamente de manera contemporánea, en el año 2010, comenzó la actividad de ADC en torno al aborto. La organización apoyó diversas acciones de La Campaña y elaboró documentos con argumentos jurídicos sobre el aborto junto con otras personalidades y organismos.

["En ADC en el 2010, ahi fue cuando Paola Bergallo y el grupo de ellas nos transfieren insumos con la propuesta de que desde ADC empecemos a trabajar el tema y hacer incidencia, intervenir en acciones judiciales (...). Paralelamente, apoyamos como las diversas acciones de La Campaña, sacamos una posición sobre despenalización, la necesidad de despenalizar y legalizar, una posición nuestra con argumentos jurídicos"] (Entrevista a F. Rossi, exintegrante de ADC). 
Medici, Ch. Estrategias para el aborto legal: la incidencia internacional. Argentina (2000-2017). Derecho y Ciencias Sociales. Mayo - Octubre 2020. № 23. Pgs 70-92 ISNN 1852-2971 Instituto de Cultura Jurídica y Maestría en Sociología Jurídica. FCJ y S. UNLP

La organización comenzó a participar en conjunto con otros actores mediante la presentación de Informes Alternativos o Informes Sombra ${ }^{8}$. En los años siguientes tuvo una importante participación en litigios a nivel interno, sobre todo en el caso "F.A.L.", que se detalla más adelante, en el que coordinó la presentación de amicus curiae tanto ante Corte Suprema como ante instancias inferiores ${ }^{9}$. Luego tuvo una destacada intervención en las judicializaciones de los protocolos sanitarios dictados luego de la sanción del fallo.

Más adelante otras organizaciones como el Equipo Latinoamericano de Justicia y Género (ELA), la Fundación para el Estudio de la Mujer (FEIM) y Amnistía Internacional Argentina se sumaron a la incidencia internacional. Una gran variedad y cantidad de organizaciones participó también de la redacción de documentos presentados a los Comités ${ }^{10}$.

\section{Las principales acciones de incidencia internacional}

\subsection{Los litigios internacionales}

Una de las acciones más importantes realizadas en el ámbito internacional fue el litigio ante el Comité de Derechos Humanos de Naciones Unidas del caso "L.M.R vs. Argentina", una joven discapacitada que requirió junto con su madre la interrupción de su embarazo producto de un abuso sexual. Este fue llevado adelante por varias organizaciones profesionalizadas pertenecientes a La Campaña, el Instituto de Género, Derecho y Desarrollo (INSGENAR), CLADEM y Católicas por el Derecho a Decidir (CDD), que la acompañaron junto con su madre a través de diversos obstáculos burocráticos interpuestos por el personal estatal.

En su sentencia del año 2011 el organismo consideró que se violaron los artículos 3 y 17 del Pacto Internacional de Derechos Civiles y Políticos, es decir, el derecho a la igualdad y el derecho

\footnotetext{
${ }^{8}$ Algunos pueden consultarse en ELA et al. (2010) y ADC et al. (2012).

${ }^{9}$ Tanto sectores promotores como detractores del aborto legal intervinieron en el caso que culminó en la Corte y procuraron, respectivamente, ampliar o restringir el derecho lo más posible. La organización ADC coordinó la estrategia de los 14 "amicus curiae" presentados por los primeros ante la Corte, quienes requirieron que se confirmara la decisión del Superior Tribunal de Chubut. Participaron de ellos CELS, CLADEM, INSGENAR, CLACAI, la Secretaría de Derechos Humanos del Ministerio de Justicia de la Nación, Human Rights Watch, Women's Link Worldwide y personalidades de la medicina como Aníbal Faúndez, entre otros. Por otra parte, un conjunto de organizaciones católicas nacionales y del continente americano presentaron 30 de estas acciones para solicitar que el fallo se revocara. Las organizaciones más eminentes fueron la Asociación Portal de Belén, Americans United for Life, Alliance Defense Fund, profesionales de la medicina, del derecho y profesores/as de la Pontifica Universidad Católica de Chile, la Universidad Católica de Valparaíso, la Universidad de los Andes y la Universidad Rey Juan Carlos de Madrid y expertos/as en bioética de estas casas de estudio, la Universidad Austral y la diputada Cynthia Hotton.

${ }^{10}$ Muchas de estas organizaciones pueden identificarse en los Informes referidos en este trabajo, como ELA et al. (2010) y ADC et al. (2012), ANDHES et al. (octubre de 2016) y ANDHES et al. (3 abril de 2017)
} 
Medici, Ch. Estrategias para el aborto legal: la incidencia internacional. Argentina (2000-2017). Derecho y Ciencias Sociales. Mayo - Octubre 2020. No 23. Pgs 70-92 ISNN 1852-2971 Instituto de Cultura Jurídica y Maestría en Sociología Jurídica. FCJ y S. UNLP

a no ser objeto de injerencias arbitrarias en la vida privada. También indicó que se violó el artículo 7, es decir, el derecho a no ser sometido a tratos crueles, inhumanos o degradantes, al serle negada la interrupción de un embarazo producto de una violación ("L.M.R vs Argentina", 2011).

En el sistema interamericano se emitió otro fallo muy importante, "Caso Artavia Murillo y Otros ('Fecundación in Vitro') vs. Costa Rica”, que trató la prohibición de la fertilización in vitro por parte del gobierno del país centroamericano. Esa decisión había partido del entendimiento de que el procedimiento era contrario al art. 4.1 de la Convención Americana de los Derechos Humanos, referenciado más arriba.

La Comisión Interamericana de los Derechos Humanos ya había fallado en los años 80 en un caso conocido como "Baby Boy" ("Baby Boy", 1981) en el que indicó que la frase fue incorporada en un nivel muy general para que el resguardo al que se refiere sea acorde con las leyes de los países que permiten la práctica en algunos casos. El criterio fue reiterado por la Corte Interamericana en el fallo "Caso Artavia Murillo y Otros ('Fecundación in Vitro') vs. Costa Rica" del año 2012, en el que afirmó que existe "un reconocimiento internacional y comparado del concepto de protección gradual e incremental de la vida en la etapa prenatal" (párr. 256), lo que dio por tierra con uno de los principales argumentos de los sectores detractores del derecho. Organizaciones como ADC presentaron amicus curiae en el proceso.

\subsection{La incidencia en los comités de las Naciones Unidas y la Organización de los Estados} Americanos.

Otra línea de trabajo importante fue la incidencia sobre los comités de las Naciones Unidas. Estos organismos son las voces más autorizadas sobre la interpretación de los tratados internacionales, y se celebran sesiones periódicas sobre la aplicación de estos en cada uno de los estados miembros, a los que acuden representantes del Estado y de la Sociedad Civil, presentando respectivos informes. El resultado son documentos que guían al Estado acerca de cómo accionar sobre determinados temas ${ }^{11}(\mathrm{ONU}, \mathrm{s} / \mathrm{f})$.

Diversas organizaciones se involucraron en la presentación de informes sombra que incluyeron varios puntos sobre el aborto en las reuniones de estos comités, los cuales respondieron con la emisión de observaciones y recomendaciones al respecto. Trabajaron conjuntamente ONG muy

\footnotetext{
${ }^{11}$ Para más información visitar UNESCO (s/f).
} 
Medici, Ch. Estrategias para el aborto legal: la incidencia internacional. Argentina (2000-2017). Derecho y Ciencias Sociales. Mayo - Octubre 2020. No 23. Pgs 70-92 ISNN 1852-2971 Instituto de Cultura Jurídica y Maestría en Sociología Jurídica. FCJ y S. UNLP

reconocidas con otras menos famosas y algunas entidades estatales, como la Comisión Provincial por la Memoria ${ }^{12}$.

A partir del nuevo milenio, gracias a este trabajo de incidencia, se multiplicaron las resoluciones que primero llaman la atención sobre la escasa aplicación del aborto no punible, para años después incluso aconsejar la despenalización.

En el año 2010, el Comité de Derechos Humanos, en sus Observaciones finales para Argentina, expresó su preocupación por la legislación restrictiva en la materia, así como también por la inconsistente interpretación de los tribunales de las causales de no punibilidad contenidas en el art. 86, y solicitó al Estado que capacitara al funcionariado acerca de los alcances de dicha norma, y que modificara la normativa de tal forma que ayude a las mujeres a evitar embarazos no deseados y abortos clandestinos que ponen en riesgo su vida (Comité de Derechos Humanos, 2010, párr. 13).

En 2010 el Comité de los Derechos del Niño, en sus Observaciones finales para nuestro país, expresó su preocupación por el elevado porcentaje de mortalidad materna vinculada con los riesgos derivados de la clandestinidad, principalmente en lo que concierne a adolescentes, y por los prolongados procedimientos para el acceso a las intervenciones legales en caso de violación. Recomendó al Estado argentino que implementara medidas urgentes para reducir estas muertes, para que el personal médico conociera la ley y efectuase la práctica sin judicialización - especialmente en caso de abuso sexual- y que enmendase el art. 86 a fines de prevenir la disparidad en su aplicación (Comité de los Derechos del Niño, 2010, párrs. 58 y 59).

En ese mismo año, el Comité para la Eliminación de la Discriminación contra la Mujer (CEDAW) en sus Observaciones finales para Argentina señaló que el acceso a servicios de salud sexual y reproductiva seguía constituyendo un grave problema para las argentinas. Expresó su preocupación por las elevadas tasas de embarazo adolescente y de mortalidad materna, la tercera parte de las cual se relaciona con el aborto clandestino. Instó al Estado a que revisara la legislación vigente que lo penaliza, dado que tiene graves consecuencias para la vida y salud de las mujeres, y también a que adoptara disposiciones necesarias para mitigar la cantidad estas muertes (Comité para la Eliminación de la Discriminación contra la Mujer, 2010, párrs. 37 y 38).

\footnotetext{
12 Los documentos correspondientes a las sesiones del Examen Periódico Universal, así como algunos otros disponibles en la página web del Alto Comisionado de las Naciones Unidas para los Derechos Humanos atestiguan la actividad conjunta de incidencia de las organizaciones entrevistadas y otras. La organización CLADEM presenta también un listado de las acciones de incidencia internacional llevadas adelante por su organización, en conjunto con muchas otras.
} 
Medici, Ch. Estrategias para el aborto legal: la incidencia internacional. Argentina (2000-2017). Derecho y Ciencias Sociales. Mayo - Octubre 2020. No 23. Pgs 70-92 ISNN 1852-2971 Instituto de Cultura Jurídica y Maestría en Sociología Jurídica. FCJ y S. UNLP

En el año 2011, el Comité de Derechos Económicos, Sociales y Culturales repitió las mismas preocupaciones expresadas por el Comité CEDAW. Recomendó al Estado adoptar todas las medidas requeridas para garantizar el acceso a la práctica cuando es legal y a prestaciones destinadas a reducir riesgos previos y posteriores a ella, a fin de disminuir el número de estos decesos (Comité de Derechos Económicos, Sociales y Culturales, 2011, párr. 22).

En 2012 el Comité sobre los Derechos de las Personas con Discapacidad se refirió a la legislación interna relativa al aborto. Lamentó que a las personas con estas condiciones que solicitan la práctica se les requiera el consenso de su representante legal para acceder a esta, y recomendó que se modificara el art. 86 para que fueran ellas quienes den su consentimiento informado valiéndose de un sistema de apoyos, de acuerdo con lo fijado en la convención (Comité sobre los Derechos de las Personas con Discapacidad, 2012, párr. 31 y 32). Un conjunto de organizaciones, entre ellas REDI y ADC, habían señalado este punto en un informe alternativo conjunto que presentaron $^{13}$.

En el ámbito interamericano se llevaron adelante sesiones del Comité de Expertas del Mecanismo de Seguimiento de la Implementación de la Convención de Belém do Pará (MESECVI) en el año 2006 (Comisión Interamericana de Mujeres, 2006), para las cuales CLADEM presentó un contrainforme (CLADEM, 2016). Se realizaron dos audiencias temáticas de la Comisión Interamericana de los Derechos Humanos, la primera de ellas en el año 2007, sobre los Derechos reproductivos de las mujeres en las Américas, con la participación del Centro por la Justicia y el Derecho Internacional (CEJIL), Human Rights Watch y el Centro de Derechos Reproductivos (CDR). En el año 2011 se llevó adelante la segunda, sobre los Derechos reproductivos de las mujeres en América Latina y el Caribe, con la intervención de ADC y otras organizaciones ${ }^{14}$.

Los anteriores documentos fueron emitidos antes del célebre fallo "F.A.L." de la Corte Suprema, que cerró numerosos debates y ofreció un marco par la intervención estatal en materia de aborto legal, que se analizará en el próximo apartado.

\footnotetext{
${ }^{13}$ Puede consultarse el informe alternativo de REDI et al. (2012).

14 También participaron el PROMSEX, Mesa por la Vida y la Salud de las Mujeres, Grupo de Información y Reproducción Elegida (GIRE), IPAS Centroamérica, Campaña 28 de Septiembre, Agrupación Ciudadana por la Despenalización del Aborto Terapéutico, Ético y Eugenésico; el CDM, el Centro de Documentación y Estudios (CDE), Colectiva Mujer y Salud, Colectiva por el Derecho a Decidir, Comissao de Cidadania e Reproducao (CCR), MYSU, Mujer y Salud.
} 
Medici, Ch. Estrategias para el aborto legal: la incidencia internacional. Argentina (2000-2017). Derecho y Ciencias Sociales. Mayo - Octubre 2020. № 23. Pgs 70-92 ISNN 1852-2971 Instituto de Cultura Jurídica y Maestría en Sociología Jurídica. FCJ y S. UNLP

Luego del fallo "F.A.L." la participación de las organizaciones en las sesiones de los comités continuó $^{15}$. Los organismos emitieron nuevas observaciones y recomendaciones relativas al aborto en Argentina. En 2016, el Comité de Derechos Humanos (CCPR) expresó satisfacción con la decisión "F.A.L." de la Corte Suprema, pero llamó una vez más la atención sobre escasa uniformidad territorial de su aplicación, la falta de protocolos y el ejercicio de la objeción de conciencia, que constituyen obstáculos de facto.

Reiteró su inquietud por los altos índices de muerte materna relacionada con el aborto clandestino y de embarazo adolescente, haciendo referencia a los arts. 3, 6, 7 y 17 del Pacto ${ }^{16}$. Recomendó revisar la legislación sobre aborto, incluso la criminal, para contemplar excepciones adicionales y asegurar que las barreras legales y las obstrucciones de hecho no obliguen a las mujeres a recurrir a abortos clandestinos.

Se refirió también al "caso de Belén", joven tucumana sentenciada en primera instancia a 8 años de prisión tras sufrir un aborto espontáneo, que tuvo muy importante trascendencia en los medios y que más adelante fue absuelta por la Corte provincial, tras una gran campaña en la que participaron organizaciones feministas y de Derechos Humanos. Indicó también repensar su situación a la luz de los estándares internacionales en la materia, con miras a su inmediata liberación, y considerar la descriminalización de la práctica (Comité de Derechos Humanos, 2016, párrs. 11 y 12).

En el año 2017, el Comité de la CEDAW expresó preocupación por el estancamiento de la tasa de mortalidad materna, debido parcialmente al aborto clandestino, por el limitado acceso a la práctica cuando es legal, a la frecuente negativa del personal médico de realizar estas intervenciones por principios éticos o religiosos y a los casos de enjuiciamiento de mujeres que interrumpieron sus embarazos. Instó al Estado a que iniciara procedimientos para que todas las provincias aprobasen protocolos en consonancia con el fallo de la Corte, velaran por que las mujeres tuviesen acceso a servicios de aborto legal y de atención posaborto, aplicaran requisitos para evitar el uso general de la objeción de conciencia por parte del personal sanitario y

\footnotetext{
${ }^{15}$ Las organizaciones presentaron importantes documentos conjuntos para las sesiones. Pueden consultarse en Abogados y Abogadas del NOA en Derechos Humanos y Estudios Sociales (ANDHES) et al. (3 de abril de 2017) y ANDHES et al. (octubre de 2016).

${ }^{16} \mathrm{El}$ art. 3 del "Pacto Internacional de Derechos Civiles y Políticos" consagra la igualdad de hombres y mujeres en el goce de todos los derechos civiles y políticos. El art. 6 indica que el derecho a la vida es inherente a la persona humana. El art. 7 sanciona el derecho a no ser sometido a torturas ni a penas o tratos crueles, inhumanos o degradantes. El art. 17 refiere al derecho a no ser objeto de injerencias arbitrarias o ilegales en la vida privada, familia, domicilio, correspondencia ni ataques ilegales a su honra y reputación.
} 
Medici, Ch. Estrategias para el aborto legal: la incidencia internacional. Argentina (2000-2017). Derecho y Ciencias Sociales. Mayo - Octubre 2020. N²3. Pgs 70-92 ISNN 1852-2971 Instituto de Cultura Jurídica y Maestría en Sociología Jurídica. FCJ y S. UNLP

acelerasen la aprobación de proyectos de ley que incluyan además casos de incesto y malformaciones fetales incompatibles con la vida extrauterina (Comité para la Eliminación de la Discriminación contra la Mujer, 2016, párrs. 32 y 33 b, c y d).

En el año 2018 el Comité de los Derechos del Niño volvió a referirse al aborto, y recomendó al Estado argentino que garantice el acceso de las adolescentes a servicios de aborto sin riesgo y atención post aborto que aseguren que la opinión de la interesada será escuchada, en atención a las altas tasas de embarazo adolescente y muerte por aborto (Comité de los Derechos del Niño, 2018, párr. 32).

Ese mismo año el Comité de los Derechos Económicos, Sociales y Culturales llamó la atención sobre los numerosos abortos riesgosos y la relación de estos con la mortalidad materna, las dificultades para acceder al aborto legal como la falta de acceso a medicinas requeridas o la objeción de conciencia de los médicos. Lamentó que no se haya aprobado la despenalización del aborto.

Recomendó el establecimiento de un marco legal para el acceso al aborto a fin de reducir el número de muertes maternas evitables, así como para evitar que la objeción de conciencia obstruya el acceso al derecho la adopción de medidas para la implementación en todas las provincias del fallo "F.A.L.”, la aprobación de protocolos en todas las jurisdicciones, la provisión de instalaciones, suministros y servicios para satisfacer las necesidades de atención previos y posteriores al aborto, la toma de medidas necesarias para garantizar el acceso a misoprostol y mifepristona, la liberalización las condiciones para el aborto legal y la no criminalización a las mujeres que recurran al aborto (párr. 56). También solicitó al Estado que en los 24 meses siguientes a la aprobación de estas observaciones provea información sobre la aplicación de las recomendaciones formuladas, entre otras cuestiones, sobre el aborto legal (párr. 68).

Respecto al ámbito Interamericano, en el año 2012 se llevaron adelante audiencias temáticas de la CIDH sobre el "Acceso a la información pública y derecho a la salud sexual y reproductiva en las Américas", con la participación de $\mathrm{ADC}^{17}$. En el año 2013 se realizó una audiencia sobre "Derechos reproductivos y contracepción de emergencia en las Américas", con la participación de CLADEM, CDD y otras organizaciones (CLADEM et al., 29 de octubre de 2013); otra sobre los "Derechos Humanos y criminalización del aborto en Sudamérica" (ADC et al., 15 de marzo

\footnotetext{
${ }^{17}$ También participaron Center for Reproductive Rights, PROMSEX, Mesa por la Vida y la Salud de las Mujeres, GIRE, Colectiva por el Derecho a Decidir, Planned Parenthood Federation of America (PPFA).
} 
Medici, Ch. Estrategias para el aborto legal: la incidencia internacional. Argentina (2000-2017). Derecho y Ciencias Sociales. Mayo - Octubre 2020. No 23. Pgs 70-92 ISNN 1852-2971 Instituto de Cultura Jurídica y Maestría en Sociología Jurídica. FCJ y S. UNLP

de 2013); y una sobre la "Situación de derechos humanos de las mujeres en Argentina", con la única concurrencia del Estado argentino.

En 2014 se llevó adelante una audiencia temática de la CIDH sobre los derechos sexuales y reproductivos de las mujeres de las Américas, a partir de la iniciativa de CDD, CLADEM y otras organizaciones (CLADEM et al., 30 de octubre de 2014).

En 2016 se llevó adelante una audiencia temática de la CIDH sobre los derechos sexuales y reproductivos de las mujeres en América del Sur, con la participación de Amnistía Internacional y Women's Link Worlwide e IPAS (2016); y una sobre Derechos Humanos, Estado laico y morbimortalidad femenina en las Américas ${ }^{18}$.

\section{4. $\quad$ El fallo "F.A.L." y su vínculo con la incidencia internacional}

En el año 2012 se sancionó el dictamen "F.A.L. s/ medida autosatisfactiva" ("F.A.L., s/ medida autosatisfactiva”, 2012), que reafirmó la decisión del Superior Tribunal de Chubut que autorizaba el aborto legal a ser practicado sobre una joven no discapacitada que gestaba producto de un abuso sexual. La sentencia recogió los debates y criterios que se fueron estableciendo los años anteriores mediante los dictámenes de los comités. Cerró definitivamente la posibilidad de realizar algunas lecturas del artículo 86 al indicar que, de acuerdo con los principios de igualdad y prohibición de toda discriminación, es irrazonable la interpretación según la cual sería legal la práctica solamente si la víctima es discapacitada (párr. 15 del fallo). Subrayó que obligar a una mujer a llevar a término una gestación que es el producto de una violación a sus derechos más fundamentales sería contrario al principio de dignidad de las personas ${ }^{19}$, del que se desprende que estas deben ser entendidas como un fin en sí mismo, y que además proscribe que sean tratadas

\footnotetext{
${ }^{18}$ Participaron de esta audiencia CDC, PROMSEX, Taller de Comunicación Mujer, Red Latinoamericana y del Caribe de Personas Trans (REDLACTRANS), Asociación Líderes en Acción, Centro Popular Para América Latina de Comunicación (CEPALC), United Belize Advocacy Movement, Organización TRANS Reinas de la Noche (OTRANS), Venezuela Diversa, Campaña por una Convención Interamericana de Derechos Sexuales y Reproductivos, Coalición de Organizaciones LGBTTTI con trabajo en la OEA, Iniciativas Globales por los Derechos Humanos, Akahatá Equipo de Trabajo en Sexualidades y Géneros, Argentina, Making Sex \& Gender Diversity Part of T\&T's National Identity, Trinidad \& Tobago, Letra S, Sida, Cultura y Vida Cotidiana, A.C., México D.F., Liga Brasileira de Lésbicas (LBL), Brasil, Taller de Comunicación Mujer, República Dominicana, Ovejas Negras, Uruguay, Alfil - Identidades en Diálogo, Ecuador, Transfamilias, Colombia, Colectivo de Sororidad, Ecumenismo y Democracia (SED), Colombia, Corporación Bogotana para el Avance de la Razón y el Laicismo, Colombia. La información sobre la audiencia está disponible en la página web de la OEA.

${ }^{19}$ Estos derechos están consagrados en el artículo 11 de la Convención Americana sobre Derechos Humanos, en el artículo $1^{\circ}$ de la Declaración Universal de los Derechos Humanos y en los preámbulos del Pacto Internacional de Derechos Civiles y Políticos y de la Declaración Americana de los Derechos y Deberes del Hombre.
} 
Medici, Ch. Estrategias para el aborto legal: la incidencia internacional. Argentina (2000-2017). Derecho y Ciencias Sociales. Mayo - Octubre 2020. No 23. Pgs 70-92 ISNN 1852-2971 Instituto de Cultura Jurídica y Maestría en Sociología Jurídica. FCJ y S. UNLP

utilitariamente (párr. 16 del fallo). Por lo tanto, la exigencia de requisitos no señalados por la ley para acceder a la interrupción del embarazo constituye, según la Corte Suprema, una práctica contra legem en la que un poder del Estado actúa interfiriendo en el acceso a los derechos y garantías constitucionales y convencionales en lugar de garantizarlos (párrafos 19 y 23).

La sentencia, asimismo, fijó la interpretación de tratados y convenciones internacionales y de la Constitución Nacional que ocasionaron debates. Indicó que el art. 75 inc. 23 de la Constitución se creó con miras de dictar un marco normativo relativo a la seguridad social y no uno punitivo, y por lo tanto no puede ser sustento de la penalización (párr. 9). Recordó que en la Convención Constituyente de 1994 hubo un amplio debate sobre el derecho a la vida, sin que hubiera intenciones de limitar el art. 86 (párr. 9). Señaló que no se deduce, ni del art. 1 de la Declaración Americana de los Derechos y Deberes del Hombre ni del art. 4 de la Convención Americana sobre Derechos humanos, la prohibición del aborto en estos casos, dado que estas normas fueron formuladas especialmente de tal forma que no se derivara de ellas la invalidez de la despenalización o legalización de la práctica en algunas circunstancias ${ }^{20}$ (párr. 10 del fallo). Definió también que el artículo 86 se armoniza con el Sistema Interamericano de Derechos Humanos de acuerdo con lo consagrado en sus Tratados y Convenciones ${ }^{21}$ (p. 117).

El fallo también subraya las múltiples veces que los diversos comités de la ONU respaldaron la legalidad del aborto en determinados casos y hace referencia, entre otros, a los documentos detallados más arriba. El Comité de Derechos Humanos se manifestó a favor de que se permitiera la práctica en las circunstancias que contempla el art. 86 (Comité de Derechos Humanos, 2000b, 2000c, 2004, 2010) (párr. 12 del fallo) El Comité de los Derechos del Niño sugirió de forma repetida que aquellos Estados que no contemplaran la posibilidad de interrumpir embarazos

\footnotetext{
${ }^{20}$ Respecto de esto cita el caso "Baby Boy" de la CIDH ("Baby boy", 1981).

${ }^{21}$ El fallo hace referencia a los siguientes derechos: el derecho a la vida (art. 1 de la Declaración Americana de los Derechos y Deberes del Hombre [en adelante, DADDH], 1948; art. 4.1 de la Convención Americana de los Derechos Humanos [en adelante, CADH], 1969; art. 3.a Convención Interamericana para Prevenir, Sancionar y Erradicar la Violencia contra la Mujer "Convención de Belém do Pará" [en adelante, Convención de Belém do Pará], 1994; a la salud (art. 11, DADDH, 1948, y art. 10 Protocolo Adicional a la Convención Americana en materia de Derechos Económicos, Sociales y Culturales, "Protocolo de San Salvador", 1948), a la libertad y seguridad personales (art. 1, DADDH, 1948; art. 7.1, CADH, 1969; art. 3.c, Convención de Belém do Pará, 1994), a la integridad física, psíquica y moral (art. 5.1, CADH, 1969; art. 3.b, Convención de Belém do Pará, 1994), a no ser sometido a torturas ni a penas o tratos crueles, inhumanos o degradantes (art. 5.2., CADH, 1969; art. 3.d., Convención de Belém do Pará, 1994), a la igualdad y no discriminación (art. 2, DADDH, 1948; art. 1.1 y 24, CADH, 1969; art. 3.f., Convención de Belém do Pará, 1994), a la dignidad (art. 17, DADDH, 1948; art. 11.1, CADH, 1969; y art. 3.e., Convención de Belém do Pará, 1994), a no ser objeto de injerencias arbitrarias o abusivas en su vida privada (art. 5, DADDH, 1948 ; y art. 11.2 y 11.3, CADH, 1969), y a la protección especial a la niñez (art. 7, DADDH, 1948; y art. 19, CADH, 1969). En especial, la Convención de Belém do Pará dispone que la violencia contra las mujeres comprende la violencia sexual (art. 2), consagra el derecho de las mujeres a una vida libre de violencia (art. 3), y reconoce que la violencia contra las mujeres impide y anula el ejercicio libre pleno de sus derechos civiles, políticos, económicos, sociales y culturales (art. 5) (1994).
} 
Medici, Ch. Estrategias para el aborto legal: la incidencia internacional. Argentina (2000-2017). Derecho y Ciencias Sociales. Mayo - Octubre 2020. N²3. Pgs 70-92 ISNN 1852-2971 Instituto de Cultura Jurídica y Maestría en

Sociología Jurídica. FCJ y S. UNLP

producto de una violación modificasen su legislación (Comité de los Derechos del Niño, 1999, párr. 30; 2001, párrs. 46 y 47; 2010, párr. 58 y 59.d) (párr. 13 del fallo), lo cual implica que la penalización no se deduce de la correspondiente convención. Los órganos de aplicación de los tratados incluso condenaron al Estado Argentino por no garantizar el acceso al derecho, desde el sistema de salud y sin injerencia del Poder Judicial, como sucedió en el caso "L.M.R. c/ Argentina" (2011, párr. 26; Comité de los Derechos del Niño, 2010) (párr. 26).

Consideró asimismo que la interpretación restrictiva del artículo 86 es contraria a la Convención de Interamericana para Prevenir, Sancionar y Erradicar la Violencia contra la Mujer en su artículo 7, que dicta las obligaciones del Estado respecto de la víctima de violencia, y es también plausible de ser considerada una forma de violencia institucional en términos de los arts. 3 y 6 de la ley 26.485 (Convención para la Eliminación de la Discriminación contra la Mujer, 1994, párr. 24).

Finalmente, el dictamen solicitó a las autoridades nacionales, provinciales y de la Ciudad Autónoma de Buenos Aires que implementaran protocolos hospitalarios de atención y que dispusieran de un sistema que permita al personal de salud manifestar su objeción de conciencia, a fines de remover todo tipo de demoras y barreras administrativas de acceso (párr. 26). También señaló la necesidad de que se extremen los recaudos para brindar a las víctimas de violencia sexual la asistencia adecuada para resguardar su salud e integridad física, psíquica, sexual y reproductiva y asesoramiento legal. Además, solicitó que se impulsaran campañas de difusión de estos derechos enfocadas a la población vulnerable y capacitaciones al personal sanitario desde todos los niveles de gobierno.

La sentencia constituyó el hito más importante del debate, y con ella se abrió una nueva etapa en la interpretación legal del artículo 86, dado que transformó los marcos y límites en los que puede pensarse el abordaje estatal sobre aborto en Argentina. A su vez, este suceso se enmarca en una serie de controversias y luchas políticas a nivel regional y supranacional, como se verá en el próximo capítulo.

\section{Palabras finales}

Entre las variadas formas de acción política para la legalización /liberalización del aborto, la incidencia internacional y los litigios estratégicos fueron de suma importancia. Una gran variedad 
Medici, Ch. Estrategias para el aborto legal: la incidencia internacional. Argentina (2000-2017). Derecho y Ciencias Sociales. Mayo - Octubre 2020. N²3. Pgs 70-92 ISNN 1852-2971 Instituto de Cultura Jurídica y Maestría en

Sociología Jurídica. FCJ y S. UNLP

y cantidad de organizaciones participaron, en ocasiones de manera coordinada y en otras de manera paralela, en diferentes acciones.

Fue muy importante la creación de La Campaña, que se transformó en la principal fuerza promotora de la demanda. Las organizaciones que son miembro de este colectivo participaron de acciones clave como el litigio del caso "L.M.R. vs. Argentina" en el Comité de Derechos Humanos de las Naciones Unidas. Paralelamente, el trabajo realizado por organizaciones como CEDES y más adelante CELS, ADC y otras redundó en aportes de gran relevancia.

A lo largo de los años la utilización de las instancias internacionales fue creciente y la respuesta de los comités de las Naciones Unidas y otros organismos abonó el terreno para mejorar el acceso al aborto legal. El fallo "F.A.L.", que constituyó un hito decisivo a nivel interno al crear estándares claros para el accionar estatal en la materia, hace referencia a algunos de estos documentos y al reclamo al Estado argentino en la sentencia del fallo "L.M.R vs Argentina".

Luego del fallo, continuó el trabajo de incidencia y las respuestas de los comités. La incidencia internacional dista mucho de ser la única estrategia de incidencia en las políticas públicas desarrollada por el activismo, y tampoco es la más crucial ni la más importante. Sin embargo, aunque no las resoluciones no son vinculantes para los Estados, por lo que no necesariamente tienen un impacto directo en el ámbito interno, la utilización de las instancias internacionales continúa siendo una forma importante de construcción de un marco jurídico más favorable a los derechos de las mujeres en las políticas públicas relativas a la interrupción del embarazo.

\section{Bibliografía}

Abogados y Abogadas del NOA en Derechos Humanos y Estudios Sociales (ANDHES), Asociación Católicas por el Derecho a Decidir-Argentina (CDD), Asociación Lola Mora, Centro de Estudios de Estado y Sociedad (CEDES), Centro de Estudios Legales y Sociales (CELS), Centro de la Mujer (CEDEM), Centro Intercambio y servicios Cono Sur Córdoba (CISCSA), Comité de América Latina y el Caribe para la Defensa de los Derechos de las Mujeres (CLADEM-Argentina), Equipo Latinoamericano de Justicia y Género (ELA), Fundación para Estudio e Investigación de la Mujer (FEIM), Instituto de Género, Derecho y Desarrollo (INSGENAR), Lesbianas y Feministas por la descriminalización del aborto, Mujeres por Mujeres, Mujeres Autoconvocadas de Trelew, Observatorio de Violencia de Género de la Defensoría del Pueblo de la provincia de Buenos Aires (OVG) (3 de abril de 2017). Informe conjunto. Examen Periódico Universal: Argentina. Evaluación de Tercer Ciclo. El aborto en Argentina. Recuperado de http://feim.org.ar/2017/04/03/informes-epuargentina/ 
Amnistía Internacional, Women's Link Worldwide, IPAS. (7 de junio de 2016). Derechos sexuales y reproductivos de las mujeres en América del Sur. Video recuperado de https://www.youtube.com/watch?v=CIobTDgLD2w

ANDHES, CDD, Asociación Lola Mora, CEDES, CELS, Centro de la Mujer (CEDEM), Colectivo de Investigación y Acción Jurídica de La Plata (CIAJ),Centro de Intercambios y Servicios para el Cono Sur (CISCSA-AFM), Comisión Argentina para los Refugiados y Migrantes (CAREF), CLADEM, ELA, Feministas en acción, Fundación Interamericana del Corazón (FIC), FEIM, Fundación Siglo 21, INSGENAR, Mujeres en Igualdad (MEI), Mujeres Trabajando, Observatorio de Violencia de Género de la Defensoría del Pueblo de la Provincia de Buenos Aires (octubre de 2016). Situación del aborto en Argentina. Recuperado el 22/10/2018 de http://feim.org.ar/wp-content/uploads/2016/11/cedaw2016-aborto.pdf

Artavia Murillo y Otros ("Fecundación in Vitro") vs. Costa Rica. Excepciones preliminares, fondo, reparaciones y costas. Corte Interamericana, 28 de noviembre del año 2012.

Asociación por los Derechos Civiles (ADC), Centro de Promoción y Defensa de los Derechos Sexuales y Reproductivos (PROMSEX), Women's Link Worldwide, Instituto de Estudos da Religião (ISER), Relatoría Nacional do Direito a Saude Sexual e Reprodutiva (Plataforma Dhesca Brasil) (15 de marzo de 2013). Derechos Humanos y criminalización del Aborto en Sudamérica (Audiencia). Video recuperado de https://www.youtube.com/watch?v=kEPXNAWAvLU

Baby Boy, 2141. Comisión Interamericana de Derechos Humanos, 6 de marzo de 1981.

Bergallo, P. (Comp.) (2011). Aborto y justicia reproductiva. Buenos Aires, Argentina: Editores del Puerto.

Bergallo, P. (Comp.) (2011). La liberalización del aborto: contextos, modelos regulatorios y argumentos para su debate. En Aborto y justicia reproductiva (pp. 1-54). Buenos Aires, Argentina: Editores del Puerto.

C.P.d.P., A.K. s/ Autorización. Suprema Corte de Justicia de la provincia de Buenos Aires. Recuperado de http://www.scba.gov.ar/includes/descarga.asp?id=945\&n=C100459.DOC

Campaña por el Aborto Legal, Seguro y Gratuito (s/f) Quiénes somos. Recuperado de http://www.abortolegal.com.ar/about/

Campaña por el Aborto Legal, Seguro y Gratuito (2009). Foro: Las acciones de La Campaña por el Aborto Legal, Seguro y Gratuito ante Aborto no Punible. Recuperado de http://www.abortolegal.com.ar/wp-content/uploads/2013/02/foro-aborto-no-punible2009.pdf

Campaña por el Aborto Legal, Seguro y Gratuito (2016). Presentamos el sexto Proyecto de Ley de IVE. Recuperado de http://www.abortolegal.com.ar/presentamos-el-nuevo-proyecto-deley/

Carabajal, M. (13 de octubre de 2011). Una vida en riesgo por negar un aborto. Página|12. Recuperado de http://www.pagina12.com.ar/diario/sociedad/3-178801-2011-10-13.html 
Medici, Ch. Estrategias para el aborto legal: la incidencia internacional. Argentina (2000-2017). Derecho y Ciencias Sociales. Mayo - Octubre 2020. N²3. Pgs 70-92 ISNN 1852-2971 Instituto de Cultura Jurídica y Maestría en

Sociología Jurídica. FCJ y S. UNLP

Chiarotti, S. (2006). El aborto en el marco de los derechos humanos. La situación Argentina. En S. Checa (Comp.). Realidades y coyunturas del aborto. Entre el derecho y la necesidad (pp. 91-110). Buenos Aires, Argentina: Paidós.

Código Penal Argentino. Infoleg. Información Legislativa. Recuperado de http://servicios.infoleg.gob.ar/infolegInternet/anexos/15000-19999/16546/texact.htm

Comisión Interamericana de Mujeres (2006). Reunión de Expertas/os sobre el Mecanismo de seguimiento de la Convención Interamericana para Prevenir, Sancionar y Erradicar la Violencia contra la Mujer, Convención de Belém do Pará. Acta final. Recuperado de https://www.oas.org/es/mesecvi/docs/CEVI2-Act-SP.pdf

Comité de América Latina y el Caribe para la Defensa de la Defensa de los Derechos de la Mujer (CLADEM). Contrainforme. Convención Interamericana para Prevenir, Sancionar y Erradicar la Violencia contra la Mujer. Convención de Belém do Pará. Octubre 2016. Recuperado de https://www.cladem.org/images/pdfs/informes-alternativos/argentinaes/contrainformebelendoparaargentina.pdf

Comité de América Latina y el Caribe para la Defensa de los Derechos de la Mujer (CLADEM), Estudio para la Defensa de los Derechos de la Mujer (DEMUS), Centro de Derechos Reproductivos (CDR), Centro de Promoción y Defensa de los Derechos Sexuales y Reproductivos (PROMSEX), Colectiva por el Derecho a Decidir, Planned Parenthood Federation of America (PPFA), Federación Latinoamericana de Sociedades de Obstetricia y Ginecología (FLASOG), Consorcio Latinoamericano de Anticoncepción de Emergencia (CLAE), Consorcio Internacional de Anticoncepción de Emergencia (ICEC), Centro de Derechos de Mujeres, Honduras , Frente Ecuatoriano por la Defensa de los Derechos Sexuales y Reproductivos, Ecuador, Miles por la Interrupción Legal del Embarazo (29 de octubre de 2013). Derechos reproductivos y contracepción de emergencia en las Américas (Audiencia). Video recuperado de https://www.youtube.com/watch?v=eozCenDaLeU

Comité de América Latina y el Caribe para la Defensa de los Derechos de la Mujer (CLADEM), Católicas por el Derecho a Decidir (CDC), Red de Mujeres Afrolatinoamericanas, Afrocaribeñas y de la Diáspora, Red de Salud de las Mujeres Latinoamericanas y del Caribe (RSMLAC), Enlace Continental de las Mujeres Indígenas (ECMIA), Red de Educación Popular entre Mujeres de Latinoamérica y el Caribe (REPEMLAC) (30 de octubre de 2014). Derechos sexuales y reproductivos de las Mujeres en las Américas. Video recuperado de https://www.youtube.com/watch?v=lXmuObTbTsc

Comité de Derechos Económicos, Sociales y Culturales. (14 de diciembre de 2011). E /C.12/ARG/CO/3, Examen de los informes presentados por los Estados partes en virtud de los artículos 16 y 17 del Pacto. Comité de Derechos Económicos, Sociales y Culturales. 47 período de sesiones. Recuperado de http://tbinternet.ohchr.org/_layouts/treatybodyexternal/Download.aspx?symbolno=E/C.12/A $\underline{\mathrm{RG} / \mathrm{CO} / 3 \& \mathrm{Lang}=\mathrm{En}}$

Comité de los Derechos Económicos, Sociales y Culturales. (1 de noviembre de 2018) E/C.12/ARG/CO/4. Observaciones finales sobre el cuarto informe periódico de la Argentina. Recuperado https://tbinternet.ohchr.org/_layouts/15/treatybodyexternal/Download.aspx?symbolno=CRC/ C/ARG/CO/5-6\&Lang=En 
Medici, Ch. Estrategias para el aborto legal: la incidencia internacional. Argentina (2000-2017). Derecho y Ciencias Sociales. Mayo - Octubre 2020. N²3. Pgs 70-92 ISNN 1852-2971 Instituto de Cultura Jurídica y Maestría en

Sociología Jurídica. FCJ y S. UNLP

Comité de Derechos Humanos (10 de agosto de 2016). Observaciones finales sobre el quinto informe periódico. Argentina. Nueva York. CCPR/C/ARG/CO/5. Recuperado de https://documents-ddsny.un.org/doc/UNDOC/GEN/G16/176/94/PDF/G1617694.pdf?OpenElement

Comité de Derechos Humanos (15 de noviembre de 2000a). Observaciones finales del Comité de Derechos Humanos. Argentina. $70^{\circ}$ Período de Sesiones. CCPR/CO/70/ARG. Nueva York. Recuperado http://tbinternet.ohchr.org/ layouts/treatybodyexternal/Download.aspx?symbolno=CCPR\%2 FCO\%2F70\%2FARG\&Lang=es

Comité de Derechos Humanos. (12 de agosto de 2004). Observaciones finales del Comité de Derechos Humanos. Gambia. 81 ${ }^{\circ}$ Período de sesiones. CCPR/CO/75/GMB; recuperado de http://tbinternet.ohchr.org/_layouts/treatybodyexternal/Download.aspx?symbolno=CCPR\%2 FCO $\% 2 F 75 \% 2 F G M B \& L a n g=e n$

Comité de Derechos Humanos. (15 de noviembre de 2000c). Observaciones Finales del Comité de Derechos Humanos. Perú. 70 Período de Sesiones. CCPR/CO/70/PER. Recuperado de http://tbinternet.ohchr.org/_layouts/treatybodyexternal/Download.aspx?symbolno=CCPR\%2 FCO\%2F70\%2FPER\&Lang=es

Comité de Derechos Humanos. (24 de julio de 2000b). Observaciones Finales del Comité de Derechos Humanos. Irlanda. $70^{\circ}$ Período de Sesiones. A/55/40. Recuperado de http://tbinternet.ohchr.org/_layouts/treatybodyexternal/Download.aspx?symbolno=A $\% 2 \mathrm{~F} 55$ $\% 2 \mathrm{~F} 40 \% 5 \mathrm{BVOL} . \mathrm{I} \% 5 \mathrm{D}(\mathrm{SUPP}) \& \mathrm{Lang}=\mathrm{en}$

Comité de Derechos Humanos. (31 de marzo de 2010). Observaciones finales del Comité de Derechos Humanos Observaciones finales del Comité de Derechos Humanos a Argentina en su $98^{\circ}$ período de sesiones. Nueva York, 31 de marzo de 2010. CCPR/C/ARG/CO/4; Recuperado de http://www2.ohchr.org/english/bodies/hrc/docs/CCPR.C.ARG.CO.4_S.pdf

Comité de los Derechos del Niño (21 de junio de 2010). Examen de los informes presentados por los Estados partes en virtud del artículo 44 de la Convención. CRC/C/ARG/CO/3-4. Comité de los Derechos del Niño. $54^{\circ}$ período de sesiones. Recuperado de http://www2.ohchr.org/english/bodies/crc/docs/co/CRC.C.ARG.CO.3-4_sp.pdf

Comité de los Derechos del Niño. (21 de febrero de 2001). Observaciones Finales del Comité de los Derechos del Niño. Palau. CRC/C/15/Add.149. Recuperado de http://docstore.ohchr.org/SelfServices/FilesHandler.ashx?enc=6QkG1d\%2FPPRiCAqhKb7y hsgnXZ0ChBsrwmcy8\%2F\%2BFNoDESwV7CzlklT2TCWdv3AoDQIIqX\%2Fip50s1R20R O2rrXqM3H\%2FwAFZ5WWwstvJPClmogrF0cHc8AGSHXzJCykkewh

Comité de los Derechos del Niño. Observaciones Finales del Comité de los Derechos del Niño. (24 de agosto de 1999). Chad. CRC/C/15/ Add.107. Recuperado de http://undocs.org/CRC/C/15/Add.107

Comité de los Derechos del Niño. Observaciones finales sobre los informes periódicos quinto y sexto combinados de la Argentina (1 de octubre de 2018) CRC/C/ARG/CO/5-6. Recuperado de 
Medici, Ch. Estrategias para el aborto legal: la incidencia internacional. Argentina (2000-2017). Derecho y Ciencias Sociales. Mayo - Octubre 2020. No 23. Pgs 70-92 ISNN 1852-2971 Instituto de Cultura Jurídica y Maestría en

Sociología Jurídica. FCJ y S. UNLP

https://tbinternet.ohchr.org/_layouts/15/treatybodyexternal/Download.aspx?symbolno=CRC/ C/ARG/CO/5-6\&Lang=Sp

Comité para la Eliminación de la Discriminación contra la Mujer (16 de agosto de 2010). Observaciones finales del Comité para la Eliminación de la Discriminación contra la Mujer a Argentina. $46^{\circ}$ Período de Sesiones. CEDAW/ARG/CO/6. Nueva York. Recuperado de http://www.acnur.org/t3/fileadmin/Documentos/BDL/2012/8408.pdf?view=1

Comité para la Eliminación de la Discriminación contra la Mujer (25 de noviembre de 2016). CEDAW/C/ARG/CO/7. Observaciones finales sobre el séptimo informe periódico de la Argentina. Recuperado de https://documents-ddsny.un.org/doc/UNDOC/GEN/N16/402/18/PDF/N1640218.pdf?OpenElement

Comité sobre los Derechos de las Personas con Discapacidad (19 de octubre de 2012). $\mathrm{CRPD} / \mathrm{C} / \mathrm{ARG} / \mathrm{CO} / 1$. Observaciones finales sobre el informe inicial de Argentina, aprobadas por el Comité en su octavo período de sesiones (17 a 28 de septiembre de 2012). Recuperado de

https://tbinternet.ohchr.org/_layouts/treatybodyexternal/Download.aspx?symbolno=CRPD/C /ARG/CO/1\&Lang=En

Convención Americana sobre los Derechos Humanos. San José de Costa Rica (7 al 22 de noviembre de 1969). Organización de los Estados Americanos (OEA). Recuperado de https://www.oas.org/dil/esp/tratados_b-

$\underline{32 \text { convencion_americana_sobre_derechos_humanos.htm }}$

Declaración Americana de los Derechos y Deberes del Hombre. Organización de los Estados Americanos (OEA). Bogotá (1948). Recuperado de http://www.oas.org/es/cidh/mandato/Basicos/declaracion.asp

Di Marco, G. (2011). El pueblo feminista: movimientos sociales y lucha de las mujeres en torno a la ciudadanía. Buenos Aires, Argentina: Biblos.

ELA, ADC, Foro Ciudadano de Participación por la Justicia y los Derechos Humanos (FOCO), CEDES, CLADEM, FEIM, Foro por los Derechos Reproductivos (Foro DDRR) INSGENAR, CELS, CDD (2012) Informe Conjunto de la situación de los derechos sexuales y los derechos reproductivos en Argentina. Recuperado el 22/10/2018 de http://www.ela.org.ar/a2/index.cfm?muestra\&codcontenido=937\&plcontampl=44\&aplicacio $\mathrm{n}=\operatorname{app} 187 \& \mathrm{cnl}=42 \& \mathrm{opc}=55$

Equipo Latinoamericano de Justicia y Género (ELA), Center on Housing Rights and Evictions (COHRE); Programa "Género, Sociedad y Universidad" de la Secretaría de Extensión de la Universidad Nacional del Litoral (Programa de Género), Acción por los derechos en el Noroeste (ADN), Asociación por los Derechos Civiles (ADC). (2010). Informe sombra para el Comité de la Convención para la Eliminación de Todas las Formas de Discriminación contra la Mujer, $46^{\circ}$ período de sesiones- Julio de 2010. Recuperado de http://www.ela.org.ar/a2/index.cfm?fuseaction=MUESTRA\&codcontenido=349\&plcontam $\mathrm{pl}=44 \&$ aplicacion $=$ app $187 \& \mathrm{cnl}=42 \& \mathrm{opc}=55$ 
Medici, Ch. Estrategias para el aborto legal: la incidencia internacional. Argentina (2000-2017). Derecho y Ciencias Sociales. Mayo - Octubre 2020. N²3. Pgs 70-92 ISNN 1852-2971 Instituto de Cultura Jurídica y Maestría en

Sociología Jurídica. FCJ y S. UNLP

F.A.L. s/ Medida autosatisfactiva, F.259 XLVI, Corte Suprema de Justicia de la Nación, 13 de marzo de 2012. Recuperado de http://www.cij.gov.ar/nota-8754-La-Corte-Suprema-precisoel-alcance-del-aborto-no-punible-y-dijo-que-estos-casos-no-deben-ser-judicializados.html

Faerman, R. (2008). Algunos debates constitucionales sobre aborto. Revista Lexis Nexis. Recuperado de http://www.juragentium.org/topics/latina/es/faerman.htm

Ferrante, M. (2011). Sobre la permisividad del aborto en el derecho penal argentino. En P. Bergallo (Comp.). Aborto y justicia reproductiva (pp. 363-375). Buenos Aires, Argentina: Editores del Puerto.

Iglesias, M. (4 de junio de 2015). Histórica marcha contra la violencia machista. Clarín. Recuperado de http://www.gente.com.ar/actualidad/ni-una-menos-el-grito-que-tomo-lacalle/18721.html

Irraizábal, M.G. (2015). La religión en las decisiones sobre aborto no punible en la Argentina. Revista Estudos Feministas, 23(3), 735-759. $\quad$ https://dx.doi.org/10.1590/0104$\underline{026 X 2015 v 23 n 3 p 735}$

L.M.R. contra Argentina (CCPR/C/101/d/1608/2007). Comité de los Derechos Humanos de Naciones Unidas, 28 de abril de 2011. Recuperado de http://www.csjn.gov.ar/dbei/iinews/Sentencias/CCPR-C-101-D-1608-2007-Spanish.pdf

Mario, S. y Pantelides, E.A. (2009). Estimación del aborto inducido en Argentina. Notas de Población. Año XXXV (87), 95-120. Recuperado de http://repositorio.cepal.org/handle/11362/12842

Miles de personas marcharon contra la violencia de género por tercer año consecutivo. (3 de junio de 2017). Télam. recuperado de http://www.telam.com.ar/notas/201706/191126-ni-unamenos-junio-2017.html

Motta, C. y Rodríguez, M. (2000). Mujer y justicia. El caso argentino. Serie de Informes del Fondo de Apoyo Operativo de Género de LAC No. 3. Recuperado de http://web.worldbank.org/archive/website00955A/WEB/PDF/ARGENT-2.PDF

Organización de las Naciones Unidas (ONU) (s/f) Comité de los Derechos Humanos. Vigilancia del ejercicio de los derechos civiles y políticos. Recuperado el 21/10/2018 de https://www2.ohchr.org/spanish/bodies/hrc/

Pacto Internacional de Derechos Civiles y Políticos. Asamblea General de las Naciones Unidas (23 de marzo de 1966). Oficina del Alto Comisionado de Naciones Unidas para los Derechos Humanos. Recuperado de http://www.ohchr.org/SP/ProfessionalInterest/Pages/CCPR.aspx

Paro Internacional de Mujeres: cuándo surgió y por qué Argentina tiene un rol central. (7 de marzo de 2018). Clarín. Recuperado de https://www.clarin.com/entremujeres/genero/paromujeres-paro-internacional-mujeres-8m_0_H1MEblPuz.html

Red por los Derechos de las Personas con Discapacidad (REDI), Centro de Estudios Legales y Sociales (CELS), Federación Argentina de Instituciones de Ciegos y Amblíopes (FAICA), Federación Argentina de Entidades Pro Atención a las PCD Intelectual (FENDIM), 
Medici, Ch. Estrategias para el aborto legal: la incidencia internacional. Argentina (2000-2017). Derecho y Ciencias

Sociales. Mayo - Octubre 2020. № 23. Pgs 70-92 ISNN 1852-2971 Instituto de Cultura Jurídica y Maestría en

Sociología Jurídica. FCJ y S. UNLP

Asociación por los Derechos Civiles (ADC) (2012). Comité sobre los Derechos de las Personas con Discapacidad. $8^{\circ}$ período de sesiones. Evaluación sobre Argentina. Informe Alternativo. Situación de la Discapacidad en Argentina 2008-2012. Recuperado de http://www.redi.org.ar/Documentos/Informes/Informe-alternativo-al-comite-sobre-losderechos-de-las-personas.pdf

Requerimiento de Instrucción Fiscal $N^{o} 1$ referido a la muerte de A.M.A. Recuperado de www.articulacionfeminista.org.

Szulik, D., Gogna, M., Petracci, M., Ramos, S., Romero, M., (2008). Anticoncepción y aborto en Argentina: perspectivas de obstetras y ginecólogos. Salud Pública de México, 50(1), 3239. Recuperado de http://www.scielo.org. $\mathrm{mx} / \mathrm{scielo}$.php?pid=S0036$\underline{36342008000100009 \& \text { script }=\text { sci_arttext\&tlng=en }}$

Tabbush, C., Díaz, M.C., Trebisacce, C. y Keller, V. (2016). Matrimonio igualitario, identidad de género y disputas por el derecho al aborto en Argentina. La política sexual durante el kirchnerismo (2003 - 2015). Sexualidad, Salud y Sociedad, 22 (abril), 22-55. Recuperado de http://www.scielo.br/pdf/sess/n22/1984-6487-sess-22-00022.pdf

Organización de las Naciones Unidas para la Educación, Ciencia y Cultura (UNESCO) (s/f) Mas sobre la naturaleza y el estatus de los instrumentos legales y programas. Recuperado de http://www.unesco.org/new/es/social-and-humansciences/themes/advancement/networks/larno/legal-instruments/nature-and-status/ 